\title{
Low Noise Amplifier Design with Metamorphic HEMT Technology for Radioastronomy Application
}

\author{
C. Enguehard, ${ }^{1}$ V. Armengaud, ${ }^{2}$ J. Lintignat, ${ }^{1}$ B. Barelaud, ${ }^{1}$ B. Jarry ${ }^{1}$ and L.I. Babak ${ }^{3}$ \\ 1 Xlim, University of Limoges, 123 Avenue Albert Thomas,87060 Limoges Cedex,France \\ e-mail: cedric. enguehard@xlim. fr \\ 2 Thales AlineaSpace,23 Avenue J.F. Champolion,31100 Toulouse, France \\ 3 Tomsk State University of Control Systems Radioelectronics,Tomsk, Russia
}

\begin{abstract}
In this paper, a low noise amplifier based on a feedback topology designed thanks to an original method is presented. Because of strong constraints in terms of noise performance a meticulous method has been developed to choose the optimum transistor. This circuit achieves a $0.3-1.7 \mathrm{GHz}$ bandwidth. The circuit gain is greater than $15 \mathrm{~dB}$ and the noise figure is lower than $1.5 \mathrm{~dB}$ all along the bandwidth. These performances have been reached using OMMIC DOO7IH Metamorphic HEMT (MHEMT) technology.
\end{abstract}

\section{Introduction}

The future biggest radio telescope named Square Kilometre Array is a device capable to observe multiples objects in the same time with sensitivity 50 times higher than the actual system.

This instrument is a 1 millions square meter array of antennas and for each one the signal needs to be amplified. So, in order to control the costs of this project, there is an absolute necessity to provide the cheapest amplifiers with the best noise performance all together. Therefore, many investigations have been done with different technologies such as silicon with BiCMOS or advanced CMOS[1].

This paper describes the original design method used to get an ultra low noise amplifier with MHEMT technology. The amplifier shows more than $15 \mathrm{~dB}$ gain and a noise figure lower than $1.5 \mathrm{~dB}$ up to $2 \mathrm{GHz}$.

First, the method used to choose the optimum transistor to achieve a very low noise amplifier is explained. Then, the choice of a convenient feedback, to match the specifications, thanks to a dedicated software is detailed.

Simulation and measurement results for the S-Parameters as well as the noise figure are given in the last Section.

\section{Methodology for Transistor Choice [2]}

The transistor is defined by many parameters such as, DC power consumption, number of gate fingers, width and lengh of these fingers.

In the proposed method, all the transistor parameters are set randomlly using Monte Carlo statistical method.

These parameters must satisfy a $\mathrm{M}$ factor which takes into account the noise and gain condition:

$$
M=\frac{F_{\min }-1}{1-\frac{1}{G_{\text {amax }}}}
$$

Where,

$F_{\min }$ is the minimum noise figure

$G_{\text {amax }}$ is the maximum available Gain

The $\mathrm{M}$ factor is minimum for the best compromise between noise and gain performances, of course any instable solution is instantly rejected.

Moreover, an inductor is connected to the source of the transistor in order to improve the noise performance as described below (Fig. 1).

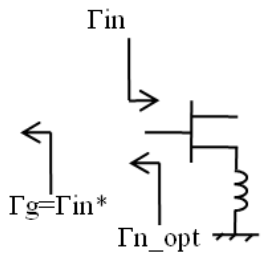

Fig. 1: Input Transistor Optimisation.

Where,

$\Gamma_{\min }$ : reflection coefficient of the input

$\Gamma_{g}$ : reflection coefficient of the optimum gain

$\Gamma_{\text {nopt }}$ : reflection coefficient of the optimum noise

With the addition of the inductor the input impedance becomes:

$$
Z_{\text {in }}=\alpha L_{\text {ind }}+j \beta L_{\text {ind }}
$$

In fact, this inductor allows to get the optimum gain reflection coefficient closer to the optimum noise reflection coefficient on the Smith chart. (Fig. 2) 


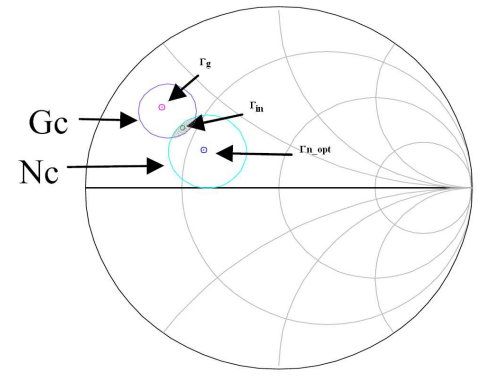

Fig. 2: Constants Gain and Noise Circles

The best inductor choice is achieved when the 2 circles are the closer.

Finally number and width of gate fingers, bias and also inductor are selected randomly and each sample is set in a graph as shown in Fig. 3.

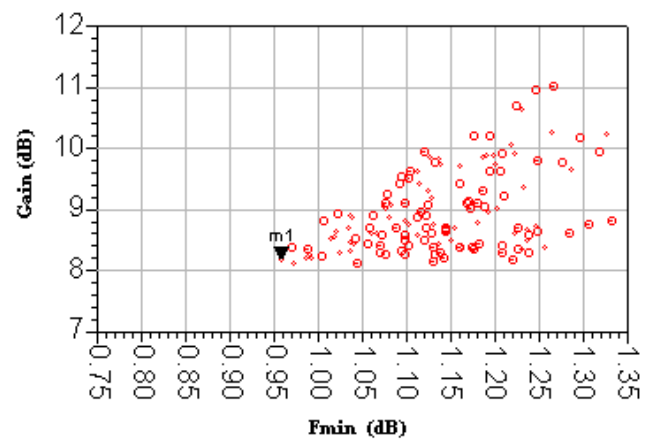

Fig. 3: Results of random choice of the transistor parameters.

The optimum transistor of the technology is obtained and corresponds to "m1" marker on Fig. 3. And its parameters are set to :

$(\mathrm{N}=8, \mathrm{~W}=75 \mathrm{~m}, \mathrm{Vgs}=0.19 \mathrm{~V}, \mathrm{Vds}=1 \mathrm{~V})$,

\section{Feedback Topology and LNA Design Assited by Software}

\subsection{Feeback Topology Choice}

A topology assistant is used [3]. Thanks to the $\mathrm{S}$ parameters file and the noise file of the transitor, it calculates the feedback impedances that are required to obtain the expected gain, noise figure and reflections coefficients.

First, it is required to select a feedback topology, here a parallel feedback.

With the S parameters and noise files the software draws the constant gain, constant noise factor and reflexion coefficient circles in a complex plan.
The expected performances are specified for each frequency (Gain, Noise, S11, S22, stability condition). Then an acceptable solution for the topology at each frequency, is defined.

The share impedance solution is the complex impedance to implement in the topology to match all the performances goals (Fig. 4).

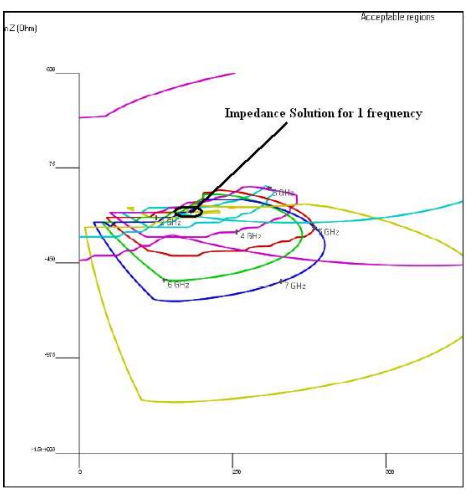

Fig. 4: Complex Impedance Solution.

This impedance depends on frequency, so it can be difficult to design a circuit which follows the frequency evolution. Therefore, a second software has been developed.

\subsection{Feedback Cell}

With the complex impedance expression at each frequency, the software suggests a panel of elementary circuits that fits the frequency evolution [4].

In this case the use of a parallel "RC" feedback appears to be convenient as can be seen on Fig. 5 .

\subsection{Circuit Design}

Then the amplifier has been designed using the optimum transistor $(\mathrm{N}=8, \mathrm{~W}=75 \mathrm{~m}, \mathrm{Vgs}=0.19 \mathrm{~V}, \mathrm{Vds}=1 \mathrm{~V})$ associated to the $\mathrm{RC}$ feedback cell $(\mathrm{R}=1.9, \mathrm{C}=4.5 \mathrm{pF})$.

Input DC-RF decoupling network is off-chip.

Figure 7 shows a picture of the LNA realized by OMMIC.

\section{Results}

Transmission gain measuremenbts are given on Fig 8 .

Some riple can be seen in the lower frequency range, to avoid this a stronger RF-DC edcoupling might have been used. 


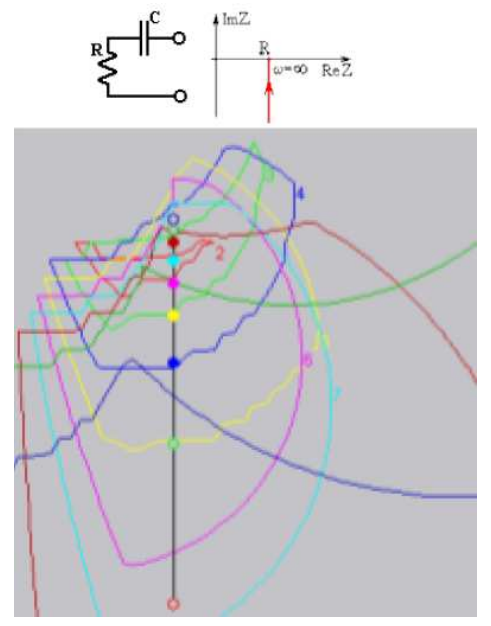

Fig. 5: Topology choice and its frequency evolution.

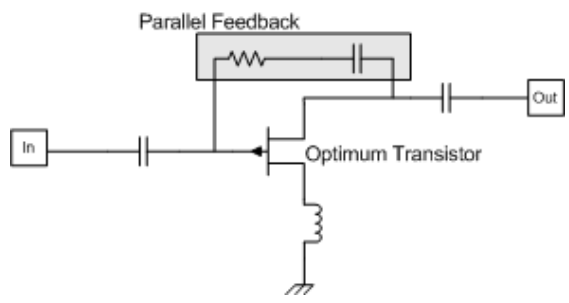

Fig. 6: Schematic of the LNA.

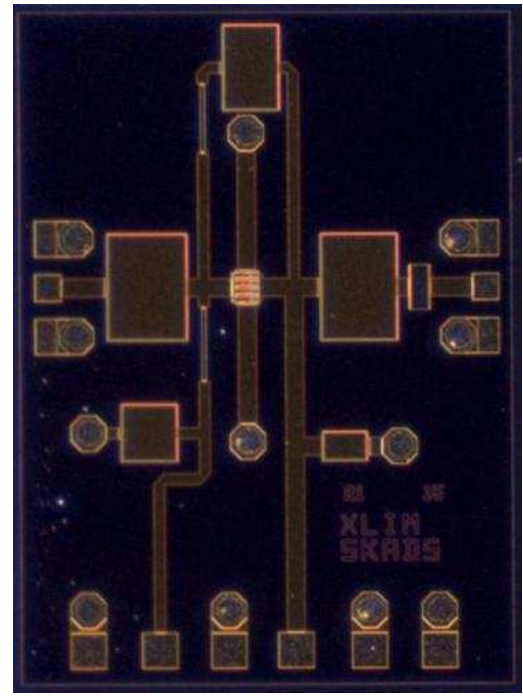

Fig. 7: Chip of the [0.3 - 1.7]GHz LNA.

The Low Noise Amplifier reachs $16.5 \mathrm{~dB}$ in the [0.3-2] $\mathrm{GHz}$ bandwidth.

The input and output reflection parameters are lower than $-11 \mathrm{~dB}$.

However, these parameters could be improved by optimazing the DC paths.

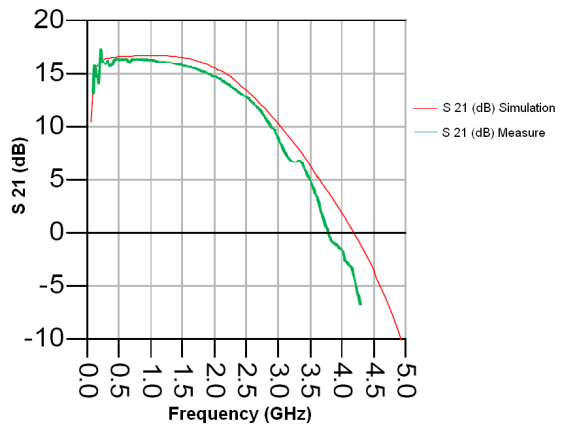

Fig. 8: Gain Performance.

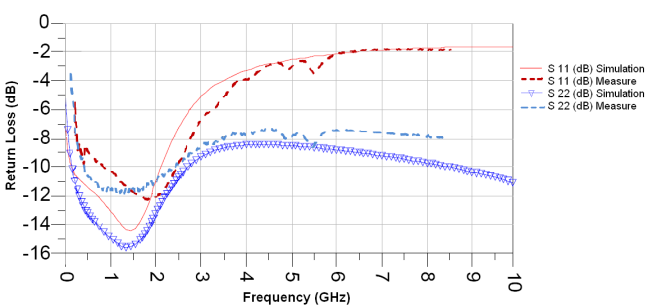

Fig. 9: Return Loss.

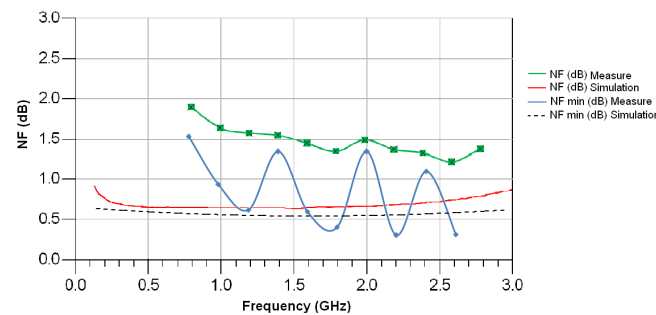

Fig. 10: Noise Figure for $Z_{0}=50 \Omega$.

The noise measurements have been performed with multiple input impedances method.

Despite the NF was expected below $1 \mathrm{~dB}$, the measured noise figure is below $1.5 \mathrm{~dB}$ all over the bandwidth.

The NFmin from the simulation is very close to the simulated NF, that is not the case for the measurements, this difference between measured NF and NFmin means that the achieved input matching is not the optimal.

\section{Conclusions}

In this paper, an original method for low noise amplifier design has been presented. This method provides original solutions for transistor choice and associated feedback computation in order to match the performances required.

The achieved circuit shows noise performances below $1.5 \mathrm{~dB}$ in the band of interest, with a Gain close to $16 \mathrm{~dB}$.The $\mathrm{DC}$ consumption is $135 \mathrm{~mW}$ for $1^{\text {st }} \mathrm{OI}$ of $-11 \mathrm{dBm}$. 
Acknowledgements. The authors would like to thanks Tomsk University and specially L.I. Babak for the software development and their collaboration.

\section{References}

[1] J.Lintignat, S.Darfeuille, B.Barelaud, L.Billonet, B.Jarry, P.Meunier, P.Gamand, "A 0.1-1.7 GHz, 1.1dB NF Low Noise Amplifier for Radioastronomy Application", IEEE Proc. Microwave Integrated Circuit Conference, pp 231-234, Oct 2007.

[2] V. Armengaud, J. Lintignat, B. Barelaud, B. Jarry, L.I. Babak, C. Laporte, "Design of a Ka-Band MMIC Filtering LNA with a Metamorphic HEMT Technology for Space Application", 38th EuMc conference, October 2008, Amsterdam.

[3] David Eyllier, M.V. Cherkachin, L.I.Babak, L.Billonnet, B.Jarry, D.A.Zaitsv, A.V.Dyagylev, " Utilisation d'une technique visuelle pour la conception d'un LNA dans la bande 2$10 \mathrm{GHz}$ ",14me Journe Nationales Microondes, 11-13 Mai 2005, Nantes.

[4] L.I. Babak, M.V. Cherkashin and A.V. Litvinov, "LOCUSA Program of Interactive Visual Design of Matching and Compensation Networks", Tomsk, 2001. 